\title{
Vítima silenciosa: violência doméstica contra o idoso no Brasil
}

\author{
Silent victims: domestic violence against elderly in Brazil
}

\author{
Carmen Silvia Molleis Galego Miziara ${ }^{1}$, Marcela Valério Braga², Fabiana \\ Iglesias de Carvalho ${ }^{1}$, Thiago Victa Teixeira ${ }^{1}$, Ivan Dieb Miziara ${ }^{3}$, \\ Daniel Romero Muñoz ${ }^{3}$
}

\begin{abstract}
Miziara CSMG; Braga MV; Carvalho FI; Teixeira TV; Miziara ID; Muñoz DR. Vítima silenciosa: violência doméstica contra o idoso no Brasil. Saúde, Ética \& Justiça. 2015;20(1):1-8.

RESUMO: O crescimento da população idosa é um fenômeno global e com ele as questões médicas e sociais têm assumido destaque. A violência contra as pessoas idosas se tornou problema de saúde pública em consequência do alto índice de morbimortalidade associada a ela. A identificação de idosos submetidos a maus-tratos é dever de todos, principalmente de médicos, para que desfechos negativos não ocorram. A divulgação de informações sobre as características das vítimas e dos agressores e dos principais tipos de violência é uma forma de manter os profissionais de saúde alertas, fato este que justifica a realização deste estudo. Foi realizado estudo descritivo transversal através de informações do Ministério da Saúde sobre as notificações de violência doméstica envolvendo pessoas acima de 60 anos de idade de ambos os sexos. O período analisado foi de janeiro de 2009 a dezembro de 2014. Os resultados do estudo mostraram que o número de notificações de violência doméstica contra o idoso aumentou progressivamente no período analisado, que a maioria das agressões ocorreu na residência das vítimas e os agressores mais frequentes foram os filhos. As mulheres foram as mais agredidas, mas os homens mostraram maior frequência de mortalidade; as agressões físicas e a negligência foram as mais relatadas. Apesar de ter havido crescimento de notificações, esses valores ainda estão distantes da realidade, em função da probabilidade de subnotificação. Os médicos precisam estar alertas quanto às possibilidades de maus-tratos e notificar os casos confirmados ou suspeitos, como determina a lei brasileira.
\end{abstract}

DESCRITORES: Maus-tratos ao idoso; Violência doméstica.

\footnotetext{
1. Faculdade de Medicina do ABC, SP, Brasil.

2. Médica Residente do Departamento de Medicina Legal, Faculdade de Medicina, Universidade de São Paulo, Brasil.

3. Faculdade de Medicina, Universidade de São Paulo, Brasil.

Endereço para correspondência: Carmen Silvia Molleis Galefo Miziara. Disciplina de Medicina Legal, Deontologia Médica, Bioética e Perícias Médicas. Departamento da Saúde da Coletividade. Faculdade de Medicina do ABC. Avenida Príncipe de Gales, 821 - Santo André. cep: 09060-650. E-mail: csmgm@uol.com.br
} 


\section{INTRODUÇÃO}

$\mathrm{C}$ om o crescimento global da população idosa, específicas questões relacionadas amplamente discutidas e dentre elas, a violênc. desempenha importante papel, pois os maus-tratos se associam ao aumento da morbimortalidade ${ }^{1,2} \mathrm{e}$, desta forma, representam grave problema de saúde pública ${ }^{3}$. A primeira publicação sobre maus-tratos contra a pessoa idosa foi em 1975 no British Scientific Journals ${ }^{4}$.

No Brasil, a necessidade de cuidar das pessoas idosas é expressa nos artigos 229 e 230 da Constituição da República Federativa do Brasil de 1988: “A família, a sociedade e o Estado têm o dever de amparar as pessoas idosas, assegurando sua participação na comunidade, defendendo sua dignidade e bem-estar e garantindo-lhes o direito à vida"5

Em 1992, durante a Assembleia Geral da Organização das Nações Unidas (ONU), foram definidos princípios que favorecem especificamente a pessoa idosa como: independência, participação, cuidados, autorrealização e dignidade 6 .

Em 1994, é sancionada a Lei $n^{\circ}$. 8.842/1994, que estabeleceu uma política nacional do idoso, e criou o Conselho Nacional do Idoso. Para efeito desta lei, idoso é a pessoa com 60 anos ou mais ${ }^{7}$.

Em 2002, outro marco da história, a Resolução $\mathrm{n}^{\mathrm{o}}$. 56.24 da Assembleia Mundial de Saúde (World Health Assembly - WHA) coloca a violência na agenda internacional como um importante problema de saúde pública em todo o mundo ${ }^{8}$

Mas foi a Lei $\mathrm{n}^{\mathrm{o}}$. 10.741/2003, que instituiu o Estatuto do Idoso, que consolidou as ações de proteção à pessoa idosa no Brasil. Esta lei também considera idosa a pessoa acima de 60 anos de idade 9 .

Segundo a Organização das Nações Unidas(ONU), a definição de idoso ainda não tem critério numérico padrão, mas é aceito o limite de 60 anos ou mais ${ }^{10}$. Maus tratos contra o idoso (abuso e negligência) são a violação dos direitos humanos individuais e civis decorrentes de ação ou omissão intencional que causa dano ou risco de dano ao idoso vulnerável por pessoa com quem ele tem relação de confiança; neste contexto, estão incluídas as condutas que não satisfazem as necessidades básicas ou a de proteção do idoso ${ }^{11}$.

O Centro Nacional sobre abuso em idosos (The National Center on Elder Abuse - NCEA) diferencia sete tipos de abuso: físico, sexual, emocional, exploração financeira, negligência, abandono e autonegligência. A definição de cada tipo de violência, conforme NCEA, é a seguinte: a) abuso físico: uso de força física que pode resultar em lesão corporal, dor física ou dano; b) abuso sexual: contato sexual não consensual de qualquer tipo com pessoa idosa; c) abuso emocional: infligir angústia, dor ou pesar através de atos verbais ou não verbais; d) exploração financeira/material: $\mathrm{O}$ uso ilegal ou indevido de fundos, bens ou ativos de uma pessoa idosa; e) negligência: recusa ou fracasso de cumprir qualquer parte de obrigações ou deveres de uma pessoa no cuidado de uma pessoa idosa; f) abandono: desamparo de idoso por um indivíduo que tem a custódia ou por uma pessoa que assumiu a responsabilidade pela prestação de cuidados do ancião; e g) autonegligência: comportamentos da pessoa idosa que ameaçam a saúde ou a segurança dele $^{12}$. De acordo com o Instituto Brasileiro de Geografia e Estatística (IBGE), em 1950 a população idosa compreendia 4\% da população total no país; em 1991, passou a representar $7,3 \%$ e, em 2000 , esta porcentagem subiu para $8.6 \%$, representando 14,5 milhões de idosos. Em 2010 , a porcentagem passa para $10 \%$ da população total ${ }^{13-}$ 15. Segundo a Organização Mundial de Saúde (OMS), o Brasil tem apresentado rápido crescimento em número e em porcentagem de pessoas idosas e, assim, necessita de novos ajustes político-sociais para enfrentar essa nova realidade ${ }^{16}$. A legislação brasileira, Lei $n^{\circ}$. 12.461/2011 ${ }^{17}$ determina que as notificações sejam obrigatórias em casos confirmados ou suspeitos de abuso contra a pessoa idosa. Para o propósito da Lei, a violência é qualquer ação ou omissão que cause a morte ou dano físico ou psicológico à pessoa idosa (acima de 60 anos de idade) ${ }^{17}$. A importância desse estudo consiste em descrever os dados mais recentes sobre as notificações de violências domésticas que acometem idosos e, assim, determinar os fatores de risco para que políticas públicas possam ser desenvolvidas e implantadas. A divulgação desta realidade, que muitas vezes não é denunciada, pode favorecer o reconhecimento de maus-tratos por profissionais que atendem os idosos evitando, desta forma, a recorrência dos atos violentos e promovendo a redução dos índices de morbimortalidade.

\section{OBJETIVO}

O objetivo desse estudo foi descrever o perfil das notificações de violência contra a pessoa idosa de acordo com os dados do Ministério da Saúde do Brasil entre janeiro de 2009 e dezembro de 2014.

\section{MÉTODOS}

Trata-se de estudo descritivo transversal, conduzido por análise de dados disponibilizados no site oficial do Ministério da Saúde do Brasil - Sistema de Informações e Agravos de Notificação - SINAN Net (http://dtr2004. saude.gov.br/sinanweb/index.php). Os critérios de inclusão foram: notificações de violência doméstica publicadas no período compreendido entre janeiro de 2009 e dezembro de 2014 envolvendo pessoas de ambos os sexos e com idade igual ou superior a 60 anos. As variáveis estudadas foram: sexo e idade das vítimas; vínculo entre a vítima e 
o agressor (conjugal formal ou informal, filho(a), amigo, desconhecido, cuidador); o tipo e a forma de violência (física, sexual, financeira/econômica, psicológica, negligência, outras); local da ocorrência da agressão e a mortalidade relacionada diretamente com a violência. Foram também anotadas informações sobre raça, nível de escolaridade e repetitividade da violência. Foram excluídas as notificações em que o sexo da vítima não constava no banco de dados. Os resultados foram comparados com a literatura médica.

\section{RESULTADOS}

No período entre janeiro de 2009 e dezembro de 2014 foram notificados no Sinan 720.730 casos de violência doméstica envolvendo pessoas de todas as idades. Neste estudo, foram excluídas 478 notificações, pois nelas não havia determinação do sexo. Das 720.252 notificações restantes, $239.934 \quad(33,3 \%)$ evolveram homens e 480.318 (66,7\%) mulheres, com 14.421 mortes relacionadas diretamente à violência, das quais 10.247 $(71,06 \%)$ foram de homens e 4.174 de mulheres. O total de notificações de agressões contra a pessoa idosa (maior ou igual a 60 anos) foi 38.895 casos no período, consistindo de 17.876 homens $(45,96 \%)$ e 21.019 mulheres $(54,04 \%)$, resultando em 1.644 óbitos masculinos (70,8\%) e 480 femininos $(29,2 \%)$ decorrentes diretamente da violência (Gráfico 1).

\section{GRÁFICO 1 - Total de notificações no período entre 2009 e 2014 de acordo com o sexo e o número de óbitos relacionados às agressões}

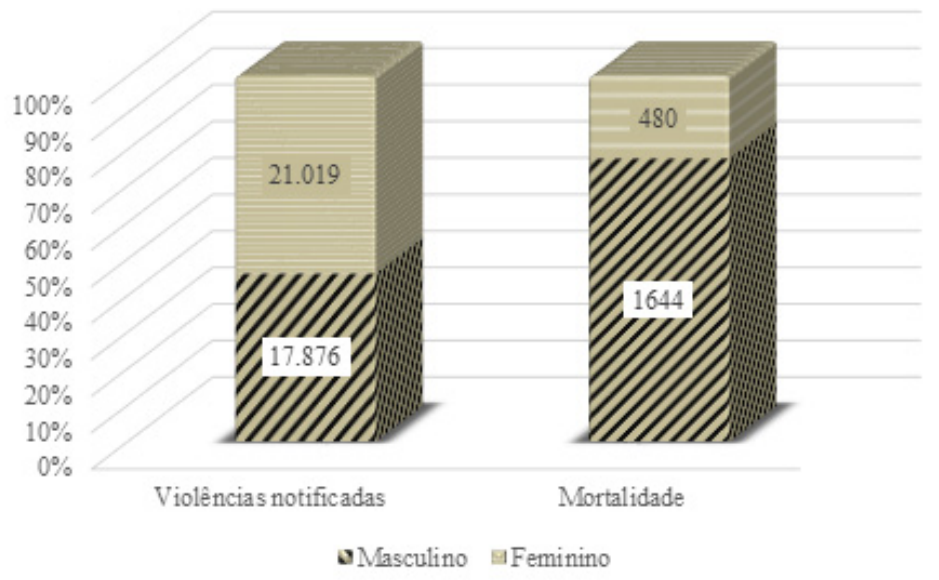

Houve predomínio da raça branca, em relação às demais, de idosos com baixo nível de escolaridade e em 13.709 notificações foram relatadas agressões repetidas e, destas, 4.679 envolviam homens e 9.030 mulheres, com 115 mortes decorrentes de agressão de repetição (75 homens e 40 mulheres).

A violência física foi a forma mais relatada em ambos os sexos vitimados e o grupo foi constituído por 12.491 homens e 12.019 mulheres, totalizando 24.510 agressões. A violência piscomoral predominou dentre as mulheres idosas (7.358 casos) em relação ao sexo masculino (2.961 notificações). As violências sexual e financeira/econômica também foram mais frequentes no sexo feminino, 792 e 1.914, respectivamente. A negligência foi a forma aplicada de abuso em 4.151 idosos e 6.110 idosas. Alguns idosos foram vítimas de mais de um tipo de agressão. Quanto ao local de ocorrência da agressão, a maioria ocorreu na residência da vítima, em ambos os sexos (Tabela 1).

A violência física resultou em 801 mortes, sendo
547 de homens e 254 de mulheres. A negligência/abandono foi responsável por 99 óbitos (34 homens e 65 mulheres); o abuso psicomoral, por 76 mortes (41 homens e 35 mulheres); o financeira/econômica se associou a 17 mortes (11 masculinas e seis femininas) e a violência sexual foi fatal em 762 mulheres e em 69 homens. Foram notificados 3.922 casos de lesões autoprovocadas no período, sendo que 2.120 envolveram homens. O desfecho fatal foi de $31,55 \%$ dentre os homens e $12,32 \%$ em mulheres. No período, foram notificados 1.274 enforcamentos, resultando em 542 mortes (42,54\%), 16.201 espancamentos com 220 óbitos (13,58\%); agressões com instrumento contundente foram notificadas 2.159 casos e, destes, 97 cursaram com óbito (4,49\%); com objeto perfurocortante, foram 2.969 casos e 206 mortes (6,93\%); ferimento por arma de fogo foram notificados em 1.254 casos e, destes, 253 evoluíram para a morte $(20,17 \%)$; o uso de veneno foi descrito em 1.237 casos, com 126 mortes (10,18\%). A Tabela 2 expõe a relação entre as diferentes formas e instrumentos mais relatados. 
Miziara CSMG et al. Vítima silenciosa: violência doméstica contra o idoso no Brasil.

TABELA 1 - Descreve os principais dados obtidos nas notificações e as porcentagens em relação ao total de notificações envolvendo pessoas com 60 anos ou mais

\begin{tabular}{|c|c|c|}
\hline & Masculino & Feminino \\
\hline & $\mathrm{n}(\%)$ & $\mathrm{n}(\%)$ \\
\hline \multicolumn{3}{|l|}{ Raça } \\
\hline Branca & $8.077(20,76)$ & $10.667(27,42)$ \\
\hline Preta & $1.219(3,13)$ & $1.571(4,04)$ \\
\hline Amarela & $142(0,36)$ & $161(0,41)$ \\
\hline Parda & $4.723(12,14)$ & $5.033(12,93)$ \\
\hline Indígena & $111(0,28)$ & $117(0,30)$ \\
\hline Não informado & $3.604(9,26)$ & $3.470(8,92)$ \\
\hline \multicolumn{3}{|l|}{ Escolaridade } \\
\hline Analfabeto & $1.494(3,84)$ & $2.171(5,58)$ \\
\hline $1^{\mathrm{a}}$ a $4^{\mathrm{a}}$ série incompleta & $3.197(8,22)$ & $3.865(9,93)$ \\
\hline $4^{\mathrm{a}}$ série completa & $1.043(2,68)$ & $1.164(2,99)$ \\
\hline $5^{\mathrm{a}}$ a $8^{\mathrm{a}}$ série incompleta & $1.117(2,87)$ & $1.297(3,33)$ \\
\hline Ensino fundamental completo & $569(1,16)$ & $850(2,18)$ \\
\hline Ensino médio incompleto & $276(0,71)$ & $299(0,76)$ \\
\hline Ensino médio completo & $502(1,29)$ & $718(1,84)$ \\
\hline Educação superior incompleta & $55(0,14)$ & $64(0,16)$ \\
\hline Educação superior completa & $218(0,56)$ & $303(0,78)$ \\
\hline Não informado & $10.278(26,42)$ & $10.288(26,45)$ \\
\hline Violência de repetição & $4.679(12,07)$ & $9.030(23,21)$ \\
\hline \multicolumn{3}{|l|}{ Tipo de violência* } \\
\hline Física & $12.491(32,1)$ & $12.019(30,90)$ \\
\hline Psicomoral & $2.961(7,61)$ & $7.358(18,91)$ \\
\hline Negligência e abandono & $4.151(10,78)$ & $6.110(15,70)$ \\
\hline Financeira / econômica & $920(2,36)$ & $1.914(4,93)$ \\
\hline Sexual & $69(0,17)$ & $792(2,03)$ \\
\hline \multicolumn{3}{|l|}{ Local de ocorrência** } \\
\hline Residência & $10.291(26,45)$ & $16.536(42,51)$ \\
\hline Habitação coletiva & $173(0,44)$ & $160(0,41)$ \\
\hline Bar ou similar & $494(1,27)$ & $92(0,23)$ \\
\hline Via pública & $2.664(6,85)$ & $1.368(3,51)$ \\
\hline Comércio & $624(1,60)$ & $171(0,43)$ \\
\hline Não informado ou outros & $3.909(10,05)$ & $2.692(0,92)$ \\
\hline
\end{tabular}

*Alguns idosos sofreram mais de um tipo de agressão

**Algumas agressões ocorreram em mais de um local, segundo dados das notificações

TABELA 2 - Tipos e instrumentos mais frequentes utilizados de agressão mais notificados e a porcentagem em relação ao total de agressões de pessoas com 60 anos ou mais

\begin{tabular}{lllll}
\hline & \multicolumn{2}{c}{ Total de notificações } & \multicolumn{2}{c}{ Óbitos } \\
\hline Masculino & Feminino & Masculino & Feminino \\
\hline Objeto perfurocortante & $7.548(19,40)$ & $8.653(22,24)$ & $145(0,37)$ & $75(0,19)$ \\
\hline Objeto contundente & $2.024(5,20)$ & $945(2,43)$ & $153(0,39)$ & $53(0,13)$ \\
\hline Arma de fogo & $1.362(3,50)$ & $797(2,05)$ & $77(0,19)$ & $20(0,05)$ \\
\hline Enforcamento & $953(2,45)$ & $301(0,77)$ & $222(0,57)$ & $31(0,08)$ \\
\hline Envenenamento & $716(1,84)$ & $558(1,43)$ & $423(1,09)$ & $119(0,30)$ \\
\hline
\end{tabular}

Foram reportados 10.627 filhos agressores, sendo 6.783 mães vítimas; os cônjuges responderam por 3.429 atos violentos, com 38 casos fatais. Amigos ou pessoa conhecida foram indicados como perpetradores em 3.602 casos, sendo a maioria das vítimas do sexo masculino (2.170); pessoas desconhecidas, em 4.108 notificações, predominando na vítima masculina (2.727); irmãos, em 1.066 casos; cuidadores, em 1.253 , com 17 óbitos. A Tabela 3 expõe com mais detalhes o vínculo que a vítima mantinha com o agressor. 
Miziara CSMG et al. Vítima silenciosa: violência doméstica contra o idoso no Brasil.

TABELA 3 - Vínculo entre o agressor e a vítima e a porcentagem em relação ao total de agressões de pessoas com 60 anos ou mais

\begin{tabular}{lllll}
\hline & \multicolumn{2}{c}{ Total de notificações } & Óbitos \\
\hline Filho(a) & Masculino & Feminino & Masculino & Feminino \\
\hline Desconhecido & $3.844(9,88)$ & $6.783(17,43)$ & $27(0,07)$ & $39(0,10)$ \\
\hline Amigo ou conhecido & $2.727(7,01)$ & $1.381(3,55)$ & $150(0,38)$ & $43(0,11)$ \\
\hline Cônjuge & $2.170(5,58)$ & $1.432(3,68)$ & $62(0,16)$ & $18(0,04)$ \\
\hline Cuidador & $897(2,30)$ & $2.532(6,51)$ & $14(0,03)$ & $24(0,06)$ \\
\hline Irmão(ã) & $468(1,20)$ & $785(2,02)$ & $4(0,01)$ & $13(0,03)$ \\
\hline
\end{tabular}

\section{DISCUSSÃO}

Estima-se que em 2020 a população idosa brasileira será de 32 milhões e, assim, o país ocupará a sexta posição em número de idosos no mundo $^{18}$. Juntamente com este crescimento, os problemas médicos e sociais também crescerão e, dentre eles, temos os maustratos contra a pessoa idosa.

A velhice é considerada condição de vulnerabilidade e, portanto, medidas de proteção visam a melhoria na qualidade de vida e a dignidade humana. A Lei $n^{\circ} .12 .461 / 2011^{17}$, altera o artigo 19 do Estatuto e determina a obrigatoriedade de notificação compulsória em casos suspeitos ou confirmados de violência praticada contra a pessoa idosa ${ }^{17}$.

A definição e a classificação de maus-tratos são controversas, assim como a definição de idoso (alguns estudos consideram o limite de idade de 60 anos, enquanto outros, 65 anos). A OMS propõe que, para países em desenvolvimento, o limite etário seja de 60 anos ou mais de idade, prevalecendo o mínimo de 65 anos para os países desenvolvidos ${ }^{19}$. A Organização das Nações Unidas (ONU) adota o critério de 60 anos ou mais ${ }^{20}$. Estas variações afetam as conclusões epidemiológicas, sobretudo quanto à incidência de maus-tratos contra a pessoa idosa ${ }^{21-24}$.

Estudo realizado por Cooper et al. ${ }^{1}$ (2008) mostrou que $6 \%$ das pessoas idosas pesquisadas reportaram significante abuso no último mês que precedeu a pesquisa, e 5,6\% dos casais relataram violência física no último ano que antecedeu o estudo. Um a cada quatro idosos em situação de vulnerabilidade são considerados em risco para sofrer algum tipo de abuso, entretanto, apenas poucos casos são detectados ${ }^{1}$. Mascarenhas et al. ${ }^{25}$ (2012) analisaram vários aspectos de violência contra a pessoa idosa no Brasil. Os autores descreveram que o perfil da vítima em geral é composto por pessoas brancas, do sexo masculino, entre 60 e 69 anos de idade, solteiros ou viúvos, com baixo nível educacional e com debilidade física ou mental ou comportamental. A forma de violência mais prevalente entre os homens foi a física, enquanto a psicológica, a sexual e a negligência foram mais observadas entre as mulheres ${ }^{25}$.

Algumas hipóteses tentam explicar o porquê da violência contra o idoso. A vulnerabilidade do idoso, física ou mental, a psicopatologia do agressor (transtorno mental ou dependência química), o estresse do cuidador, o ganho secundário do abusador (teoria da troca) e a violência transgeracional (internalização como fatos aceitáveis de violência vividas na infância) são algumas delas $^{21}$. No Brasil, as notificações de agressões ao idoso, sejam elas suspeitas ou confirmadas, são compulsórias e os dados são reportados ao Ministério da Saúde. A análise do número de notificações por violência doméstica de janeiro de 2009 a dezembro de 2014 mostrou um aumento bruto no decorrer do período, mas com discreta desaceleração em 2014. Os idosos submetidos à violência doméstica representaram $5,4 \%$ das agressões em todas as idades. Estudo português analisou as violências contra a pessoa idosa no período entre janeiro e junho de 2013 e concluiu que $23,5 \%$ dos idosos sofreram algum tipo de abuso, sendo que a negligência e o abuso emocional foram os mais relatados ${ }^{26}$. No Brasil, no mesmo período (janeiro a junho de 2013), 5.482 idosos foram agredidos, representando $6,02 \%$ do total de agressões notificadas naquele período. Dados do U.S. National Elder Mistreatment Study, mostram uma prevalência anual de maus-tratos contra o idoso em torno de $11.4 \%{ }^{27}$. Estes cenários podem sugerir que no Brasil ocorra a subnotificação dos maus-tratos e, provavelmente, os números mostrados no nosso estudo não refletem a realidade brasileira, posto que muitos idosos deixam de denunciar as agressões por medo de represália, por falta de condição de locomoção ou cognitiva ou porque têm vínculo afetivo ou de dependência com o agressor. Segundo Cooper, $25 \%$ dos idosos vulneráveis têm risco de abuso e apenas em uma pequena proporção esse crime é detectado ${ }^{1}$. Os filhos foram os perpetradores mais frequentemente citados nas notificações e os cônjuges ocuparam a quarta posição, ficando atrás de agressores desconhecidos e amigos. No estudo desenvolvido por Frazão et al. (2014), os cônjuges foram os agressores 
em $49 \%$ e os filhos em $47 \%{ }^{28}$ A porcentagem de idosas submetidas à violência em relação às mulheres de todas as faixas etárias foi de $4,37 \%$ e a dos idosos de $7,46 \%$, mas a comparação entre os sexos na faixa etária, maior ou igual a 60 anos, mostrou um discreto predomínio feminino $(54,04 \%)$. Esses dados mostram que as mulheres são as maiores vítimas em todas as faixas etárias. Nossos resultados são semelhantes aos descritos por Bond e Butler ${ }^{29}$ (2013) e Verdejoa e Calvo ${ }^{30}$ (2014). Dentre os tipos de violência, a física predominou em ambos os sexos, dados semelhantes aos encontrados por Frazão et al. $^{28}$ (2014). Segundo os autores, a mulheres com transtornos motores relataram terem sido submetidas a episódios prévios de abuso ${ }^{28}$. Muitas lesões físicas externas são confundidas com lesões próprias da idade, fato este que dificulta a identificação de ato não acidental. A negligência foi considerada em 10.261 notificações $(26,38 \%)$ e o abuso psicomoral em 10.319 $(26,53 \%)$, mostrando que aproximadamente um quarto das agressões sofridas pelos idosos não são fáceis de serem diagnosticadas, pois não deixam sinais físicos, mas são fatores associados a estresse e sofrimento. Estima-se que cerca de 700.000 a 1.2 milhão de idosos sofram esse tipo de agressão ${ }^{29}$. As lesões autoprovocadas foram relatadas em 3.922 notificações $(10,08 \%)$, os homens foram as principais vítimas $(54,05 \%)$. Este resultado é preocupante, pois o crescimento de lesões autoprovocadas é um fenômeno mundial. O suicídio envolvendo idosos é considerado problema de saúde pública. Estudo desenvolvido em 13 países europeus pelo Multicentre Study of Suicidal Behaviour (WHO/EURO) mostrou que as taxas médias de morte por autoviolência, entre pessoas de mais de 65 anos nessas sociedades, chega a $29.3 / 100.000^{31}$. Embora o enforcamento tenha sido notificado em 1.274 casos, $42 \%$ destas vítimas evoluíram para o óbito, em contraste com apenas $13,58 \%$ das vítimas de agressão por força corporal. As notificações femininas mostraram que houve predomínio de agressão por força corporal/espancamento e envenenamento. Os homens foram vítimas de agressões por objetos contundentes, perfurocortantes e perfurocontundentes. A porcentagem de mortalidade em homens foi superior à de mulheres em todas as formas de agressão. Esses dados mostram que, embora as mulheres sejam vítimas mais frequentes de maus-tratos, são os homens que sofrem as agressões mais graves e fatais.

\section{CONCLUSÃo}

Este estudo mostrou que o número de notificações de maus-tratos contra a pessoa idosa tem crescido, mas provavelmente as notificações ainda não expressam a realidade. As mulheres são as vítimas mais frequentes, mas a mortalidade é proporcionalmente mais elevada dentre os homens. Os agressores, na maioria dos casos, são os filhos e a forma física foi a violência mais declarada. Os idosos, por manterem vínculo afetivo ou de dependência com os perpetradores, tendem a omitir a violência, fato este que pode sugerir que as agressões sejam omitidas pelas vítimas. Portanto, os médicos precisam estar atentos a esta grave situação e é recomendado que, ao atenderem idosos vítimas, levantem sempre esta possibilidade diante de inconsistência entre as manifestações clínicas do idoso e a história médica. Essa medida pode auxiliar na identificação de abuso contra a pessoa idosa. A notificação compulsória de casos confirmados ou suspeitos de maus-tratos é estabelecida por lei e visa à proteção dos idosos.

Conflito de interesse: não há.

Miziara CSMG; Braga MV; Carvalho FI; Teixeira TV; Miziara ID; Muñoz DR. Silent victims: domestic violence against elderly in Brazil. Saúde, Ética \& Justiça. 2015;20(1):1-8.

\begin{abstract}
The increase in the elderly population is a global phenomenon and with it medical and social issues have taken on great importance. Violence against the elderly has become a public health problem due to the high rates of morbidity and mortality (M\&M) associated with it. Identifying elderly maltreatment is everyone's duty, especially the doctor's, so that negative outcomes do not occur. Disclosure of information about victim's and abuser's characteristics and about the main types of violence is a way of keeping health professionals alert, thus justifying this study. This is a transversal descriptive study based on information from Brazil's Health Ministry on domestic violence reports involving people over 60 years old of both genders. The time frame reviewed ranges from January, 2009 through December, 2014. The results of the study show that the number of reports concerning domestic violence against the elderly progressively increased in the time span referred above. Most aggressions occurred in the victim's home and oftentimes the abusers were the victim's sons. Women were more commonly abused, however men had a higher mortality frequency. Physical aggression and negligence were the most often reported. Despite the apparent rise in reports, these rates are still far from the actual rates in reality, due to under-reporting. Doctors should be aware of the possibility of abuse and report on confirmed or suspected cases, in compliance with the Brazilian laws.
\end{abstract}

KEY WORDS: Elder abuse; Domestic violence. 


\section{REFERÊNCIAS}

1. Cooper C, Selwood A, Livingston G. The prevalence of elder abuse and neglect: a systematic review. Age Ageing. 2008; 37(2):151-60. DOI: http://dx.doi.org/10.1093/ageing/ afm194

2. Dong X, Chen R, Chang ES, Simon M. Elder abuse and psychological well-being: a systematic review and implications for research and policy - a mini review. Gerontology. 2013; 59(2):132-42. DOI: http://dx.doi. org/10.1159/000341652

3. Lachs MS, Williams CS, O'Brien S, Pillemer KA, Charlson ME. The mortality of elder mistreatment. JAMA. 1998; 280(5):428-32.

4. WHO - World Health Organization. World Report on Violence and Health. Geneva: World Health Organization; 2002. p. 123-46.

5. Brasil. Constituição da República Federativa do Brasil. $1^{\mathrm{a}}$ ed. Brasília (DF): Câmara dos Deputados, Edições Câmara; 1988.

6. ONU. Resolução 46/91 - Aprovada na Assembleia Geral das Nações Unidas em 16/12/1991, trata dos direitos dos idosos. Assembleia Geral das Nações Unidas; 1991.

7. Brasil. Lei $n^{\circ} .8 .842$, de 4 de janeiro de 1994. Dispõe sobre a política nacional do idoso, cria o Conselho Nacional do Idoso e dá outras providências. Diário Oficial da União. 1994 jan 5.

8. Peshevska DJ, Markovik M, Sethi D, Serafimovska E. Relationships and Community Risk Factors for Elder Abuse and Neglect: Findings from the First National Prevalence Study on Elder Maltreatment. Maced J Med Sci. 2014; 7(2):369-74. DOI: http://dx.doi.org/10.3889/mjms.18575773.2014 .0382

9. Brasil. Lei $\mathrm{n}^{\circ} 10.741$, de $1^{\circ}$ de outubro de 2003. Dispõe sobre o Estatuto do Idoso e dá outras providências. Diário Oficial da União. 2003 out 3.

10. WHO - World Health Organization. Definition of older or elderly person [Internet]. [acesso em 2015 mar 15]. Disponível em: http://www.who.int/healthinfo/survey/ ageingdefnolder/en/

11. Bonnie RJ, Wallace RB, editors. Elder mistreatment: Abuse, neglect and exploitation in an aging America. Washington, DC: National Academies Press; 2003.

12. Tatara T, Kuzmeskus LM. Types of Elder Abuse in Domestic Settings [Internet]. National Center on Elder Abuse; 1999. [Acesso em 2015 mar 15]. Disponível em: http://www. ncea.aoa.gov/Resources/Publication/docs/fact1.pdf

13. Brasil. IBGE - Censo 2012 [Internet]. IBGE, 2012. [Acesso em 2015 mar 15]. Disponível em: http://www.ibge. gov.br/home/estatistica/populacao/perfilidoso

14. Fagundes SD, Silva MT, Thees MFRS, Pereira MG. PrevalenceofdementiaamongelderlyBrazilians:asystematic review. Sao Paulo Med J. 2011; 129(1):46-50. DOI: http:// dx.doi.org/10.1590/S1516-31802011000100009

15. WHO - World Health Organization. Envelhecimento ativo: uma política de saúde [Internet]. Pró-Saúde, 2005. [Acesso em 2015 mar 15]. Disponível em: http://www.prosaude. org/publicacoes/diversos/envelhecimento_ativo.pdf

16. WHO - World Health Organization. Global Health and Aging [Internet]. WHO, 2011. [Acesso em 2015 Mar 15].
Disponível em: http://www.who.int/ageing/publications/ global_health.pdf

17. Brasil. Lei ${ }^{\circ}$. 12.461 . Altera a Lei ${ }^{\circ} 10.741$, de $1^{\circ}$ de outubro de 2003, para estabelecer a notificação compulsória dos atos de violência praticados contra o idoso atendido em serviço de saúde. Diário Oficial da União. 2011 jul 27.

18. Veras R. Em busca de uma assistência adequada à saúde do idoso: revisão de literatura e aplicação de um instrumento de detecção precoce e de previsibilidade de agravos. Cad Saude Pública. 2003; 19(3):705-15. DOI: http://dx.doi. org/10.1590/S0102-311X2003000300003

19. Brasil. IBGE. Notas Técnicas [Internet]. IBGE, 2004. [Acesso em 2015 mai 23]. Disponível em: http://www. ibge.gov.br/home/estatistica/populacao/condicaodevida/ indicadoresminimos/sinteseindicsociais2004/notatecnica. pdf

20. ONU-BR - Nações Unidas no Brasil. A ONU e as pessoas idosas [Internet]. ONU, 2002. [Acesso em 2015 mai 23]. Disponível em: http://nacoesunidas.org/acao/pessoasidosas/

21. Giurani F, Hasan M. Abuse in elderly people: the Granny Battering revisited. Arch. Gerontol. Geriatr. 2000; 31(3):215-20.

22. WHO - World Health Organization. A global response to elder abuse and neglect: building primary health care capacity to deal with the problem worldwide: Main Report [Internet]. WHO, 2008. [Acesso em 2015 mar 15]. Disponível em: http:/www.who.int/ageing/publications/ ELDER_DocAugust08.pdf

23. Victorian Government Department of Human Services. With respect to age - 2009: Victorian Government practice guidelines for health services and community agencies for the prevention of elder abuse [Internet]. Melbourne, 2009. [Acesso em 2015 Mar 15]. Disponível em: http://www. health.vic.gov.au/agedcare/downloads/ wrta/with_respect to_age.pdf

24. Akaza K, Bunai Y, Tsujinaka M, Nakamura I, Nagai A, Tsukata $\mathrm{Y}$, et al. Elder abuse and neglect: social problems revealed from 15 autopsy cases. Leg Med (Tokyo). 2003; 5(1):7-14.

25. Mascarenhas MDM, Andrade SSCA, Neves ACM, Pedrosa AAG, Silva MMA, Malta DC. Violência contra a pessoa idosa: análise das notificações realizadas no setor saúde - Brasil, 2010. Ciênc saúde coletiva. 2012; 17(9):2331-41. DOI: http://dx.doi.org/10.1590/S141381232012000900014

26. Martins R, Neto MJ, Andrade A, Albuquerque C. Abuse and maltreatment in the elderly. Aten Primaria. 2014; 46(Suppl 5):206-9. DOI: http://dx.doi.org/10.1016/S02126567(14)70093-9

27. Acierno R, Hernandez MA, Amstadter AB, Resnick HS, Steve K, Muzzy W, et al. Prevalence and correlates of emotional, physical, sexual, and financial abuse and potential neglect in the United States: the National Elder Mistreatment Study. Am J Public Health. 2010; 100(2):292-7. DOI: http://dx.doi.org/10.2105/AJPH.2009.163089

28. Frazão SL, Silva MS, Norton P, Magalhães T. Domestic violence against elderly with disability. J Forensic Leg 
Med. 2014; 28:19-24. DOI: http://dx.doi.org/10.1016/j. jflm.2014.09.003

29. Bond MC, Butler KH. Elder Abuse and Neglect. Definitions, Epidemiology, and Approaches to Emergency Department Screening. Clin Geriatr Med. 2013; 29(1):257-73. DOI: http://dx.doi.org/10.1016/j.cger.2012.09.004

30. Verdejo IC, Calvo CB. Analysis of violence against elderly woman. Procedia Soc Behav Sci. 2014; 161(19):110-4. DOI: http://dx.doi.org/10.1016/j.sbspro.2014.12.018

31. De Leo D, Padoani W, Scocco P, Bille-Grahe U, Arcsnman E, Bjerke T, et al. Elderly suicidal behaviour: results from WHO/EURO Multicentre Study on Parasuicide. Int J Geriatr Psychiatr 2001;16(3):300-10. DOI: http://dx.doi. org/10.1002/gps.337

Recebido para publicação: 25/05/2015

Aceito para publicação: 23/06/2015 